MS04 Biophysical

characterization and

crystallization

Chairs: Dr. Andrzej M. Brzozowski.

Dr. Pavlina Rezacova try (also known as thermal shift assays) and dynamic light scattering.

This talk will discuss some of the older as well as newer methods for characterizing a protein sample prior to crystallization or running a binding assay. The talk will have an educational tone with the hope of stirring up discussions around protein quality and how best to assess it.

Keywords: protein analysis, biophysical methods

MS04-01

\section{Biophysical methods for analyzing protein quality}

Annette Roos ${ }^{1}$, Yasmin Andersson ${ }^{2}$

1. Biophysical Screening and Characterization Facility, Drug Discovery and Development Platform, SciLifeLab, Uppsala, Sweden

2. Protein Expression and Characterization Facility, Drug Discovery and Development Platform, SciLifeLab, Stockholm, Sweden

email: annette.roos@icm.uu.se

Science for Life Laboratory (SciLifeLab) is a Swedish national center for molecular biosciences supported by the Swedish government. The aim is to make state-of-the-art technologies and know-how available to academic researchers across the whole of Sweden. The Drug Discovery and Development (DDD) Platform is one part of the center. We help Swedish academic groups progress therapeutic ideas towards a pre-clinical proof-of-concept. Projects can have a small molecule focus or the aim can be to produce an antibody therapeutic. The platform consists of eight facilities with different areas of expertise, including assay development and high-throughput compound screening, antibody generation, protein expression, biophysical characterization, medicinal chemistry, pharmacological profiling, analysis of drug metabolism and pharmacokinetics data, and target safety analysis. For more information see https://www. scilifelab.se/platforms/ddd

At the Biophysical Screening and Characterization Facility in the DDD Platform, we characterize interactions between molecules. Our three key techniques are surface plasmon resonance (SPR), X-ray crystallography, and microscale thermophoresis (MST). Most of our DDD projects are to verify binding of compounds found through high-throughput screens. Our objective is to improve the high-throughput hits to become lead molecules.

A recurring problem within many of the projects has been the lack of a functioning reference compound to verify that the target protein behaves in an expected manner. The quality of protein samples is critical in all the techniques we offer, but the lack of reference compounds makes it even more important. The proteins we work with are either provided by the academic group we collaborate with, are bought from companies that provide such services, or are produced by our sister facility within the DDD, the Protein Expression and Characterization Facility. At the DDD we prefer the latter as here we know the protein samples will be well characterized and we can have a dialogue about producing protein in a manner suited to the needs of the project. The analysis methods we mainly use are size exclusion chromatography, various gel electrophoresis, differential scanning fluorime- 